\title{
Social, behavioral, and sleep characteristics associated with depression symptoms among undergraduate students at a women's college: a cross-sectional depression survey, 2012
}

\author{
Katherine T Wilson ${ }^{1}$, Ashley E Bohnert ${ }^{1}$, Alex Ambrose ${ }^{1}$, Destiny $Y$ Davis $^{1}$, Dina M Jones ${ }^{1,2}$
} and Matthew J Magee 1,3* $^{*}$

\begin{abstract}
Background: The association between student characteristics and depression among students attending women's colleges (single-sex institutions of higher education that exclude or limit males from admission) is poorly understood. Our objective was to estimate the prevalence of depression and determine behavioral and social characteristics associated with depression among students attending a women's college.

Methods: We administered a cross-sectional Internet-based survey between April and May 2012 to students $(n=277)$ enrolled at a private women's college in the southeastern US. Center for Epidemiologic Studies Depression (CES-D) and Depression Anxiety Stress Scale 21 (DASS-21) instruments measured self-reported depression. Bivariate and multivariable logistic regression methods were used to estimate adjusted associations.

Results: Prevalence of depression measured by CES-D and DASS-21 instruments was 26.3\% (95\% confidence interval [CI] 20.8-32.3\%) and 26.0\% (95\% Cl 20.4-32.3\%), respectively. After adjusting for confounders, absence of strong social support (prevalence odds ratio $[\mathrm{OR}]=4.3,95 \% \mathrm{Cl} 1.4-13.7$ ), history of mental health disorder (OR=4.8 95\% Cl 1.9-12.4), and poor sleep hygiene $(\mathrm{OR}=2.8,95 \% \mathrm{Cl}$ 1.3-5.8) were associated with depression.

Conclusions: This cross-sectional survey identified absence of strong social support, history of mental health disorder, and poor sleep hygiene as potential predictors of depression among students attending a women's college. Further investigation of these factors may inform depression interventions for students attending women's colleges and other undergraduate student populations.
\end{abstract}

Keywords: Mental health, Depression, Sleep, Women's college

\section{Background}

Depression is a frequently reported mental disorder in the US college student population. In the spring 2012 American College Health Association National College Health Assessment of students from 141 self-selecting postsecondary education institutions, over one-fifth of undergraduate students reported a current or previous diagnosis with depression [1]. Studies have identified

\footnotetext{
* Correspondence: mjmagee@emory.edu

'Agnes Scott College Department of Public Health, 141 East College Avenue, Decatur, USA

${ }^{3}$ Emory University Rollins School of Public Health, Department of Epidemiology, 1518 Clifton Road Northeast, Atlanta, USA

Full list of author information is available at the end of the article
}

depression among college students as a risk factor for poor academic performance [2,3], problem drinking [4], suicidal thoughts $[5,6]$, frequent illness [7], and college dropout [8].

Several risk factors for depression among US college students have been investigated, but debate remains regarding which factors are consistently associated with increased risk of depression. Studies have identified lack of certain types of exercise [9-11] and sleep quantity [12] as likely risk factors. Poor sleep quality among college students is also predictive of poor academic performance [13,14], problem drinking [15], and suicidal thoughts [16]. Among adolescents, short sleep duration is associated

\section{Biomed Central}

(c) 2014 Wilson et al.; licensee BioMed Central Ltd. This is an open access article distributed under the terms of the Creative Commons Attribution License (http://creativecommons.org/licenses/by/2.0), which permits unrestricted use, distribution, and reproduction in any medium, provided the original work is properly cited. 
with suicidality, illicit drug use, and depression defined by the Center for Epidemiologic Studies Depression (CES-D) scale [17]. Poor sleep quality and depression are likely to have a bidirectional relationship among young adults [18].

Studies among undergraduate students have reported non-significant differences between the prevalence of depression among female and male undergraduate students in the US [19-21]. However, in a national report that assessed the US prevalence of diagnosed or treated depression among college students, $12.5 \%$ of females versus $6.7 \%$ of males reported depression in the previous year ( $p$-value <0.001) [1]. Prospective observational studies among female undergraduate students have identified sleep deficit [12], lack of certain types of exercise [22], and binge eating [23] as predictors of depression.

Whether national trends in depression and risk factors for depression are similarly distributed at women's colleges (single-sex institutions of higher education that exclude or limit males from admission) remains under-investigated. Female students attending women's colleges have been found to have greater academic involvement $[24,25]$, greater self-confidence $[25,26]$, and higher satisfaction with college experience $[24,27]$ but lower satisfaction with quality of social life/interpersonal support $[27,28]$ compared to female students attending co-educational institutions. Differences in satisfaction with educational experiences at women's colleges, compared to co-educational colleges, may also affect prevalence of depression. However, few studies have assessed depression symptoms, student characteristics, or the relationship between student characteristics and depression at women's colleges [12]. Given the paucity of data on depression among women's colleges in the US, we aimed to 1) estimate the prevalence of depression symptoms among female undergraduate students at a women's college and 2) determine behavioral, social, and sleep characteristics associated with depressive symptoms among the undergraduate students.

\section{Methods}

\section{Design and data collection}

A cross-sectional survey was implemented at a private women's liberal arts college in the southeastern US. All current students, enrolled during the 2011 to 2012 academic year, were eligible to participate in the study. A 47-question, computer-based survey was administered via a commercially available Internet application between April and May 2012. All current students received three recruitment e-mails; those who consented were linked to the Internet survey. The survey could be completed from any computer with Internet connection. Participation was voluntary, and responses were anonymous.

\section{Ethics statement}

The Agnes Scott College Institutional Review Board approved the study protocol and questionnaire. Informed consent was obtained from all participants via the Internet survey tool.

\section{Measures}

The survey instrument collected self-reported information on students' demographics, individual sleep behaviors, perceptions of mental health disorders, and social network characteristics. Self-reported measures were drawn from the US Center for Disease Control's 2012 Behavioral Risk Factor Surveillance System Questionnaire [29], or were collected using single-item questions designed by the researchers. The survey also included the Center for Epidemiologic Studies Depression (CES-D) 20-item version to assess frequency of recent depressive symptoms [30]. A CES-D score was calculated for each student as previously described [30]. Current CES-D depression was defined as a score $\geq 16$ [30]. In the same Internet survey, the short form of the Depression Anxiety Stress Scale (DASS-21) 21-question assessment was also administered [31]. Current DASS-21 depression was defined as a score $\geq$ 14 as previously described [31].

\section{Statistical analysis}

Data collected via the Internet survey tool were automatically stored in an electronic database. All statistical analyses were performed using SAS Version 9.3 (SAS Institute Inc., Cary, NC). Bivariate analyses were used to determine the association between individual characteristics and current CES-D and DASS-21 depression. For bivariate analyses, the Cochran-Mantel-Haenszel chisquare test was used to calculate p-values for categorical variables, and the Student's t-test (for normally distributed) or Wilcoxon-Mann-Whitney rank sums test (for non-normally distributed) was used for continuous variables. A two-sided p-value $<0.05$ was considered statistically significant throughout the analyses. Student characteristics associated with current depressive symptoms (defined by CES-D and DASS-21) with a p-value < 0.2 were entered in predictive binomial multivariable logistic regression models using backward and stepwise elimination with a $\mathrm{p}$-value $<0.05$ required for retention. A multivariable logistic causal (non-elimination procedure) model was also used to estimate the adjusted association between sleep quality and current depressive symptoms. Confounders included in the causal model were chosen based on observed associations in the collected data, directed acyclic graph theory [32], and previous literature.

\section{Results}

During the spring semester of 2012, 305 of 820 (37.2\%) students consented to the survey. Among students who 
consented, 22 (7.2\%) were removed because they did not begin the survey, $6(2.0 \%)$ were ineligible because they were not enrolled in the undergraduate program, and 277 (90.8\%) were included in the analyses. Among the included participants, 240 (85.7\%) completed the CES-D and $227(81.1 \%)$ completed the DASS-21 portions of the survey instrument.

Most participants were residential students (80.7\%), U. S.-born (87.1\%), and non-Hispanic African American/ Black (29.1\%) or non-Hispanic White (51.5\%). The mean and median ages were 21.4 (standard deviation [SD] 5.0) and 20.4 years (interquartile range [IQR] 2.2), respectively. Class representation was approximately equal, with at least one-fifth of participants representing each class. The majority of participants (96.1\%) were enrolled as full-time students, taking at least 12 credit hours.

Among 240 participants who completed the CES-D portion of the survey instrument, 63 (26.3\%, 95\% CI 20.8$32.3 \%$ ) had a score $\geq 16$, indicating current depression (Table 1). The mean and median CES-D scores were 12.4 (SD 9.8) and 10 (IQR 10), respectively. Of 227 participants who completed the DASS-21 portion of the survey instrument, 59 (26.0\%, 95\% CI 20.4-32.3\%) had a score $\geq 14$, indicating current depression. Participants had a mean DASS-21 score of 9.5 (SD 9.6) and median DASS-21 score of 6 (IQR 12). Depression by CES-D and DASS-21 had 83.6\% agreement (Kappa value 0.57, p-value $<0.001$ ).

Self-reported overall health, family history of depressive and/or anxiety disorders, and history of clinical diagnosis or treatment for a mental health disorder were significantly associated with current depression by both

Table 1 Distribution of CES-D/DASS-21 depression among students attending a women's college, 2012

\begin{tabular}{|c|c|}
\hline Mental Health Scale & Univariate statistic \\
\hline \multicolumn{2}{|l|}{ CES-D score $(\mathrm{N}=240)$} \\
\hline Mean (SD) & $12.4(9.8)$ \\
\hline Median (IQR) & $10(10)$ \\
\hline Range & 54 \\
\hline Percent $\geq 16$ score $\left(\right.$ Exact $95 \%$ Cl) ${ }^{a}$ & $26.3 \%(20.8-32.3 \%)$ \\
\hline \multicolumn{2}{|l|}{ DASS-21 score $(\mathrm{N}=227)$} \\
\hline Mean (SD) & $9.5(9.6)$ \\
\hline Median (IQR) & $6(12)$ \\
\hline Range & 42 \\
\hline Percent $\geq 14$ score $(\text { Exact } 95 \% \mathrm{Cl})^{b}$ & $26.0 \%(20.4-32.3 \%)$ \\
\hline Percent agreement & $83.6 \%$ \\
\hline Kappa statistic ${ }^{c}$ & $0.57, p$-value $<0.001$ \\
\hline \multicolumn{2}{|c|}{$\begin{array}{l}\text { Note. }{ }^{a} \text { CES-D score } \geq 16 \text { is defined as moderate depressed mood by } \\
\text { Radloff [30]. } \\
\text { bASS- } 21 \text { score } \geq 14 \text { is defined as moderate to extremely severe depression by } \\
\text { Lovibond and Lovibond [31]. } \\
{ }^{c} \text { Assessed agreement between moderately depressed mood by CES-D } \\
\text { and DASS- } 21 .\end{array}$} \\
\hline
\end{tabular}

CES-D and DASS-21 scales (Table 2). Self-reported body mass index (BMI) and average weeknight hours of sleep were significantly associated with CES-D and DASS-21 depression by the Wilcoxon-Mann-Whitney test. Both the self-reported number of social support group members and absence of strong social support group were significantly associated with depression by both scales.

The absence of a self-reported strong social support group (adjusted odds ratio [AOR] 4.3, 95\% CI 1.4-13.7) and previous history of a mental health disorder (AOR 4.8, 95\% CI 1.9-12.4) remained significant in the backward and stepwise elimination models for current depression by CES-D (Table 3). The absence of a self-reported strong social support group (AOR 5.8, 95\% CI 1.3-25.7) and previous history of a mental health disorder (AOR 5.8, 95\% CI 1.9-17.7) also remained significant in the DASS-21 backward and stepwise elimination models. In addition, selfreported employment (AOR 4.8, 95\% CI 1.4-15.5) and overall health (AOR 5.6, 95\% CI 1.1-29.4) remained significantly associated with DASS-21 depression.

In separate non-elimination multivariable models, poor sleep quality was significantly associated with both CES-D and DASS-21 measures of depression after adjusting for confounders (Table 4). Students who reported poor or extremely poor sleep quality were more likely to also report prevalent CES-D scores $\geq 16$ (AOR 2.8, 95\% CI 1.3-5.8). Similarly, the odds of prevalent DASS-21 depression symptoms among students with poor or extremely poor sleep quality was 2.8 (95\% CI 1.4-5.8) times that of students who self-reported sleep to be excellent, good, or okay.

\section{Discussion}

In our cross-sectional survey, $26 \%$ of undergraduate students attending a women's college self-reported prevalent depression by both CES-D $(\mathrm{n}=240)$ and DASS-21 $(n=227)$ instruments. Previous history of a mental disorder and the absence of a self-reported strong social support group remained significant predictors of depression in backward and stepwise elimination models for depression by both instruments. In adjusted causal models, students with poor sleep quality had approximately 2.8 times the odds of depression (by both CES-D and DASS21) compared to that of students who reported higher quality sleep.

Previous studies among female US college students have reported the prevalence of current depression to be between $16.6 \%$ and $41.3 \%$ [21,33,34], indicating our results from a women's college are within the range of results from other co-ed institutions. Regestein et al. [12] and Herman et al. [34] reported mean CES-D scores from surveys of female college students of 17.9 (SD 10.9) and 15.4 (SD 9.7), respectively, both higher than our observed mean CES-D score of 12.4 (SD 9.8). 
Table 2 Association between CES-D/DASS-21 depression with individual behavioral, social, and community characteristics

\begin{tabular}{|c|c|c|c|c|}
\hline \multirow[b]{5}{*}{ Participant Characteristic } & \multicolumn{4}{|c|}{ Measure of depression } \\
\hline & \multicolumn{2}{|c|}{ CES-D Score } & \multicolumn{2}{|c|}{ DASS-21 Score } \\
\hline & $\geq 16^{\mathrm{a}}$ & $<16^{\mathrm{a}}$ & $\geq 14^{\mathrm{a}}$ & $<14^{\mathrm{a}}$ \\
\hline & N (\%) & N (\%) & N (\%) & N (\%) \\
\hline & $63(26.3)$ & $177(73.8)$ & $59(26.0)$ & $168(74.0)$ \\
\hline \multicolumn{5}{|l|}{ Age } \\
\hline Mean (STD) & $21.4(5.0)$ & $20.9(2.7)$ & $21.5(5.2)$ & $20.9(2.7)$ \\
\hline Median (IQR) & $20.3(2.2)$ & $20.4(1.8)$ & $20.2(2.4)$ & $20.4(1.8)$ \\
\hline $17-18$ & $4(6.7)$ & $13(8.0)$ & $4(7.2)$ & $9(5.8)$ \\
\hline $19-20$ & $34(56.7)$ & $92(56.8)$ & $32(58.2)$ & $87(56.5)$ \\
\hline $21-22$ & $17(28.3)$ & $49(30.3)$ & $12(21.8)$ & $52(33.8)$ \\
\hline$>22$ & $5(8.3)$ & $8(4.9)$ & $7(12.7)$ & $6(3.9)$ \\
\hline \multicolumn{5}{|l|}{ Class year ${ }^{c, d}$} \\
\hline Freshman & $22(34.9)$ & $40(22.7)$ & $18(30.5)$ & $32(19.5)$ \\
\hline Sophomore & $15(23.8)$ & $55(31.3)$ & $16(27.1)$ & $52(31.7)$ \\
\hline Junior & $12(19.0)$ & $51(29.0)$ & 11(18.6) & $50(30.5)$ \\
\hline Senior & $14(22.2)$ & $30(17.0)$ & $14(23.7)$ & $30(18.3)$ \\
\hline \multicolumn{5}{|l|}{ Race/ethnicity } \\
\hline African American/Black (non-Hispanic) & $15(25.0)$ & $51(29.7)$ & $13(23.6)$ & $49(29.9)$ \\
\hline Asian & $5(8.3)$ & $20(11.6)$ & $34(61.8)$ & $81(49.4)$ \\
\hline Hispanic/Latina & $3(5.0)$ & $7(4.1)$ & $3(5.5)$ & $7(4.3)$ \\
\hline White (non-Hispanic) & $35(58.3)$ & $85(49.4)$ & $2(3.6)$ & 19 (11.6) \\
\hline Multiracial/ethnic & $2(3.3)$ & $9(5.2)$ & $3(5.5)$ & $8(4.9)$ \\
\hline \multicolumn{5}{|l|}{ Nation of birth ${ }^{c, d}$} \\
\hline United States & $60(95.2)^{*}$ & $150(85.2)$ & $56(94.9)$ & $144(86.2)$ \\
\hline Other & $3(4.8)$ & $26(14.8)$ & $3(5.1)$ & $23(13.8)$ \\
\hline \multicolumn{5}{|l|}{ Residence $^{c}$} \\
\hline On-campus & $47(74.6)$ & $148(83.6)$ & $46(78.0)$ & $138(82.1)$ \\
\hline Off-campus & $16(25.4)$ & $29(16.4)$ & $13(22.0)$ & $30(17.9)$ \\
\hline \multicolumn{5}{|l|}{ GPA } \\
\hline $3.6-4.0$ & $21(33.9)$ & $64(36.6)$ & $14(24.1)$ & $63(37.7)$ \\
\hline $3.1-3.5$ & $22(35.5)$ & $75(42.9)$ & $27(46.6)$ & $69(41.3)$ \\
\hline $2.6-3.0$ & $12(19.4)$ & $24(13.7)$ & $11(19.0)$ & $22(13.2)$ \\
\hline $2.1-2.5$ & $7(11.3)$ & $12(6.9)$ & $6(10.3)$ & $13(7.8)$ \\
\hline \multicolumn{5}{|l|}{ Number of credit hours $^{d}$} \\
\hline Mean (SD) & $15.4(4.0)$ & $16.1(3.0)$ & $15.3(4.1)$ & $16.1(3.0)$ \\
\hline Median (IQR) & $16.0(2.0)$ & $16.0(2.0)$ & $16.0(2.0)$ & $16.0(2.0)$ \\
\hline $1-11$ & $4(6.8)$ & $5(3.0)$ & $4(6.8)$ & $5(3.0)$ \\
\hline $12-16$ & $38(64.4)$ & $98(58.7)$ & $40(67.8)$ & $98(58.3)$ \\
\hline$>16$ & $17(28.8)$ & $64(38.3)$ & $15(25.4)$ & $65(38.7)$ \\
\hline
\end{tabular}


Table 2 Association between CES-D/DASS-21 depression with individual behavioral, social, and community characteristics (Continued)

\begin{tabular}{|c|c|c|c|c|}
\hline \multirow[b]{5}{*}{ Participant Characteristic } & \multicolumn{4}{|c|}{ Measure of depression } \\
\hline & \multicolumn{2}{|c|}{ CES-D Score } & \multicolumn{2}{|c|}{ DASS-21 Score } \\
\hline & $\geq 16^{a}$ & $<16^{\mathrm{a}}$ & $\geq 14^{a}$ & $<14^{a}$ \\
\hline & N (\%) & N (\%) & N (\%) & N (\%) \\
\hline & $63(26.3)$ & $177(73.8)$ & $59(26.0)$ & $168(74.0)$ \\
\hline \multicolumn{5}{|l|}{ Employment $^{d}$} \\
\hline On-campus & $27(42.9)$ & $79(44.9)$ & $21(35.6)$ & $76(45.5)$ \\
\hline Off-campus & $11(17.5)$ & $36(20.5)$ & $9(15.3)$ & $37(22.2)$ \\
\hline None & $25(39.7)$ & $61(35.7)$ & $29(49.2)$ & $54(32.3)$ \\
\hline \multicolumn{5}{|l|}{ Self-reported overall health } \\
\hline Excellent/very good/good ${ }^{c, d}$ & $47(74.6)^{*}$ & $166(94.9)$ & $44(77.2)^{*}$ & $157(93.5)$ \\
\hline Fair/poor & $16(25.4)$ & $9(5.1)$ & $13(22.8)$ & $11(6.5)$ \\
\hline \multicolumn{5}{|l|}{$\mathrm{BMI}^{\mathrm{d}}$} \\
\hline Mean (SD) & $26.6(7.8)^{*}$ & $24.1(6.0)$ & $27.1(7.7)^{*}$ & $24.0(5.9)$ \\
\hline Median (IQR) & $24.7(8.4)$ & $20.6(6.1)$ & $25.3(9.2)$ & $23.0(6.0)$ \\
\hline$<18.5$ & $5(7.9)$ & $13(7.3)$ & $3(5.1)^{*}$ & $8(4.9)$ \\
\hline $18.5-24.9$ & $29(46.0)$ & $101(57.1)$ & $23(39.0)$ & $99(60.7)$ \\
\hline$>24.9$ & $29(46.0)$ & $63(35.6)$ & $33(55.9)$ & $56(34.4)$ \\
\hline \multicolumn{5}{|l|}{ Nightly hours of sleep ${ }^{c, d}$} \\
\hline Mean (SD) & $6.0(1.6)^{*}$ & $6.7(1.3)$ & $6.0(1.6)^{*}$ & $6.7(1.3)$ \\
\hline Median (IQR) & $6.0(2.0)$ & $7.0(1.0)$ & $6.0(2.6)$ & $7.0(1.0)$ \\
\hline$<5$ & $12(19.1)^{*}$ & $17(9.6)$ & $7(11.9) *$ & $5(3.0)$ \\
\hline $5-6$ & $32(50.8)$ & $67(37.9)$ & $32(54.2)$ & $69(41.8)$ \\
\hline$>6$ & $19(30.2)$ & $93(52.5)$ & $20(33.9)$ & $91(55.2)$ \\
\hline \multicolumn{5}{|l|}{ Sleep quality c,d } \\
\hline Excellent/good/okay & $35(59.3)^{*}$ & $138(82.6)$ & $35(59.3)^{*}$ & $140(83.3)$ \\
\hline Poor/extremely poor & $24(40.7)$ & $29(17.4)$ & $24(40.7)$ & $28(16.7)$ \\
\hline \multicolumn{5}{|c|}{ Family member with previous mental health diagnosis ${ }^{c, d}$} \\
\hline Depressive or anxiety disorder & $9(20.9)^{*}$ & $38(27.9)$ & $13(30.2)^{*}$ & $34(24.8)$ \\
\hline Both & $22(51.2)$ & $30(22.1)$ & $21(48.8)$ & $33(24.1)$ \\
\hline Neither & $12(27.9)$ & $68(50.0)$ & $9(20.9)$ & $70(51.1)$ \\
\hline \multicolumn{5}{|l|}{ College loans $^{d}$} \\
\hline Yes & $44(69.8)$ & $113(63.8)$ & $44(74.6)$ & $107(63.7)$ \\
\hline No & $19(30.2)$ & $64(36.2)$ & $15(25.4)$ & $61(36.3)$ \\
\hline \multicolumn{5}{|l|}{ Major } \\
\hline Undeclared & $4(7.0)$ & $7(4.5)$ & $3(5.2)$ & $7(4.5)$ \\
\hline Humanities & $11(19.3)$ & $20(13.0)$ & $10(17.2)$ & $22(14.1)$ \\
\hline Social sciences & $21(36.8)$ & $66(42.6)$ & $24(41.4)$ & $63(40.4)$ \\
\hline Natural sciences & $9(15.8)$ & $40(25.8)$ & $9(15.5)$ & $40(25.6)$ \\
\hline Double major & $11(19.3)$ & $18(11.6)$ & $11(19.0)$ & $20(12.8)$ \\
\hline Dual-degree Nursing & $1(1.8)$ & $4(2.6)$ & $1(1.7)$ & $4(2.6)$ \\
\hline \multicolumn{5}{|c|}{ Self-reported increase in stress from college } \\
\hline Yes & $53(89.8)$ & $138(83.6)$ & $53(89.8)$ & $142(84.5)$ \\
\hline No & $6(10.2)$ & $27(16.4)$ & $6(10.2)$ & $26(15.5)$ \\
\hline
\end{tabular}


Table 2 Association between CES-D/DASS-21 depression with individual behavioral, social, and community characteristics (Continued)

\begin{tabular}{|c|c|c|c|c|}
\hline \multirow[b]{5}{*}{ Participant Characteristic } & \multicolumn{4}{|c|}{ Measure of depression } \\
\hline & \multicolumn{2}{|c|}{ CES-D Score } & \multicolumn{2}{|c|}{ DASS-21 Score } \\
\hline & $\geq 16^{\mathrm{a}}$ & $<16^{\mathrm{a}}$ & $\geq 14^{\mathrm{a}}$ & $<14^{\mathrm{a}}$ \\
\hline & N (\%) & $\mathrm{N}(\%)$ & N (\%) & N (\%) \\
\hline & $63(26.3)$ & $177(73.8)$ & $59(26.0)$ & $168(74.0)$ \\
\hline \multicolumn{5}{|c|}{ Frequency of weekly academic stress ${ }^{b}$} \\
\hline Often & $44(74.6)^{*}$ & $72(43.1)$ & $46(78.0)^{*}$ & $70(41.7)$ \\
\hline Not very often & $12(20.3)$ & $80(47.9)$ & 11(18.6) & $82(48.8)$ \\
\hline Almost never & $3(5.1)$ & $15(9.0)$ & $2(3.4)$ & $16(9.5)$ \\
\hline \multicolumn{5}{|c|}{ Number of people in social support group $p^{c, d}$} \\
\hline Mean (SD) & $5.1(4.2)^{*}$ & $6.5(5.3)$ & $4.6(4.0)^{*}$ & $6.7(5.2)$ \\
\hline Median (IQR) & $4.0(4.0)$ & $5.0(5.0)$ & $3.5(3.5)$ & $5.0(4.0)$ \\
\hline $0-4$ & $30(54.5)$ & $62(38.3)$ & $35(62.5)^{*}$ & $56(34.6)$ \\
\hline $\begin{array}{l}\quad>4 \\
\text { Strong social support group } \\
\text { c,d }\end{array}$ & $25(45.5)$ & $100(61.7)$ & $21(37.5)$ & $106(65.4)$ \\
\hline Yes & $27(58.7)^{*}$ & $128(89.5)$ & $25(53.2)^{*}$ & $134(92.4)$ \\
\hline No & $19(41.3)$ & $15(10.5)$ & $22(46.8)$ & $11(7.6)$ \\
\hline \multicolumn{5}{|l|}{ Relationship status ${ }^{c}$} \\
\hline Single & $42(68.9)$ & $96(54.9)$ & $34(59.6)$ & $92(55.4)$ \\
\hline Relationship & $19(31.1)$ & $79(45.1)$ & $23(40.4)$ & $74(44.6)$ \\
\hline \multicolumn{5}{|l|}{ Children } \\
\hline Yes & $2(3.2)$ & $6(3.4)$ & $2(3.4)$ & $6(3.6)$ \\
\hline No & $61(96.8)$ & $169(96.6)$ & $57(96.6)$ & 162(96.4) \\
\hline \multicolumn{5}{|l|}{ 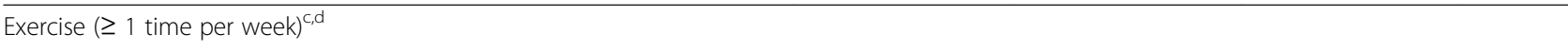 } \\
\hline Yes & $58(93.5)$ & $174(98.3)$ & $55(94.8)$ & $166(98.8)$ \\
\hline No & $4(6.5)$ & $3(1.7)$ & $3(5.2)$ & $2(1.2)$ \\
\hline \multicolumn{5}{|c|}{ Previous mental health clinical diagnosis or treatment ${ }^{c, d}$} \\
\hline Yes & $26(44.1)^{*}$ & $37(22.4)$ & $29(49.2)^{*}$ & $36(21.6)$ \\
\hline No & $33(55.9)$ & $128(77.6)$ & $30(50.8)$ & $131(78.4)$ \\
\hline \multicolumn{5}{|l|}{ Ever visited campus psychologist $^{d}$} \\
\hline Yes & $30(50.8)$ & $69(41.3)$ & 23(46.9) & $56(44.1)$ \\
\hline No & $29(49.2)$ & $98(58.7)$ & $26(53.1)$ & $71(55.9)$ \\
\hline \multicolumn{5}{|c|}{ Perceived stigma of mental health issues at college ${ }^{d}$} \\
\hline Strongly agree/Agree & $15(26.3)$ & $109(68.1)$ & $23(41.1)^{*}$ & $43(26.7)$ \\
\hline Disagree/Strongly disagree & $42(73.7)$ & $51(31.9)$ & $33(58.9)$ & $118(73.3)$ \\
\hline \multicolumn{5}{|c|}{ Perceived stress at current college higher than other universities } \\
\hline Yes & $18(31.0)$ & $50(30.3)$ & $19(32.2)$ & $52(31.0)$ \\
\hline No & $40(69.0)$ & $115(69.7)$ & $40(67.8)$ & $116(69.0)$ \\
\hline
\end{tabular}

*p-value $<0.05$.

Notes. ${ }^{a}$ May not sum to $\mathrm{N}$ because some participants did not complete this measure.

${ }^{\mathrm{b}}$ Not included in predictive model due to collinearity.

Included in predictive model for CES-D.

${ }^{\mathrm{d}}$ Included in predictive model for DASS-21.

Previous studies have reported an association between depression and sleep quantity $[35,36]$ and quality [35,37]. Similar to our findings of a strong relationship between sleep quality and depression, Regestein et al.
[12] found that residential students attending a women's college who self-reported trouble sleeping and sleepiness were significantly associated with higher CES-D scores (p-values $<0.01$ ). Poor sleep quality and 
Table 3 Multivariable analysis for predictors of CES-D/DASS-21 depression

\begin{tabular}{|c|c|c|c|c|}
\hline \multirow[b]{3}{*}{ Participant characteristic } & \multicolumn{4}{|c|}{ Measure of depression } \\
\hline & \multicolumn{2}{|l|}{$\overline{\text { CES-D }}$} & \multicolumn{2}{|l|}{ DASS-21 } \\
\hline & OR $(95 \% \mathrm{Cl})$ & $\operatorname{AOR}^{a}(95 \% \mathrm{Cl})$ & OR $(95 \% \mathrm{Cl})$ & $\operatorname{AOR}^{\mathrm{a}}(95 \% \mathrm{Cl})$ \\
\hline \multicolumn{5}{|l|}{ Employment } \\
\hline Yes & - & - & 1 & 1 \\
\hline No & & & $2.1(1.0,4.4)$ & $4.8(1.4,15.5)$ \\
\hline \multicolumn{5}{|l|}{ Self-reported overall health } \\
\hline Excellent/Very good/Good & - & - & 1 & 1 \\
\hline Fair/Poor & & & $5.6(2.0,16.0)$ & $5.6(1.1,29.4)$ \\
\hline \multicolumn{5}{|l|}{ Strong social support group } \\
\hline Yes & 1 & 1 & 1 & 1 \\
\hline No & $4.5(1.7,12.1)$ & $4.3(1.4,13.7)$ & $7.1(2.5,19.8)$ & $5.8(1.3,25.7)$ \\
\hline \multicolumn{5}{|l|}{$\begin{array}{l}\text { Previous mental health clinical } \\
\text { diagnosis or treatment }\end{array}$} \\
\hline Yes & $3.8(1.8,8.0)$ & $4.8(1.9,12.4)$ & $4.9(2.3,10.3)$ & $5.8(1.9,17.7)$ \\
\hline No & 1 & 1 & 1 & 1 \\
\hline
\end{tabular}

Note. ${ }^{a}$ Adjusted models were derived from backward and stepwise elimination techniques performed in SAS version 9.3 (SAS Institute, Cary, NC). Covariates entered into the elimination algorithm are indicated in Table 2.

depression have been found to have a bidirectional relationship among youth and young adults [18]. Studies correlating depression and sleep latency and interruptions suggest that specific aspects of sleep quality may be related to depressive symptoms [35,37]. Our finding that the absence of a strong social support group is associated with depressive symptoms is also consistent with previous studies that found more CES-D defined depression among both adults and college students with lower self-perceived emotional support from family and social networks [38-40].

\section{Limitations}

This study is subject to limitations. The cross-sectional study design provides inference only to prevalent associations, without establishing temporality, between student characteristics and depressed mood. Misclassification of depression was possible because symptoms were selfreported; nonetheless, we used two extensively validated instruments to measure depression and the anonymous nature of the survey likely reduced the potential for this bias. Both CES-D and DASS-21 instruments assess shortterm depressive mood, consequently the outcome may not

Table 4 Depression symptoms and selected study participant characteristics: Multivariable results for adjusted prevalence odds of depression

\begin{tabular}{|c|c|c|c|c|}
\hline \multirow[b]{3}{*}{ Participant Characteristic } & \multicolumn{4}{|c|}{ Measure of depression } \\
\hline & \multicolumn{2}{|l|}{ CES-D } & \multicolumn{2}{|l|}{ DASS-21 } \\
\hline & OR $(95 \% \mathrm{Cl})$ & $\operatorname{AOR}^{a}(95 \% \mathrm{Cl})$ & OR $(95 \% \mathrm{Cl})$ & $\operatorname{AOR}^{a}(95 \% \mathrm{Cl})$ \\
\hline \multicolumn{5}{|l|}{ Sleep quality } \\
\hline Excellent/Good/Okay & 1 & 1 & 1 & 1 \\
\hline Poor/Extremely poor & $3.3(1.7,6.3)$ & $2.8(1.3,5.8)$ & $3.4(1.8,6.6)$ & $2.8(1.4,5.8)$ \\
\hline \multicolumn{5}{|l|}{ Residence } \\
\hline On-campus & 1 & 1 & 1 & 1 \\
\hline Off-campus & $1.7(0.9,3.5)$ & $1.9(0.8,4.7)$ & $1.3(0.6,2.7)$ & $1.3(0.5,3.3)$ \\
\hline \multicolumn{5}{|l|}{ Self-reported overall health } \\
\hline Excellent/Nery good/Good & 1 & 1 & 1 & 1 \\
\hline Fair/Poor & $6.3(2.6,15.1)$ & $3.9(1.5,10.7)$ & $4.2(1.8,10.1)$ & $2.7(1.0,7.2)$ \\
\hline
\end{tabular}

Note. ${ }^{a}$ Multivariable adjusted models included all variables reported in the table and age. Adjustment was based on previous literature and from directed acyclic graphs. 
reflect long-term associations that would result in clinical depression. Because the study population included undergraduate students attending a women's college, the generalizability of the results to co-ed colleges may be limited. In addition, because the survey was conducted at only one undergraduate women's college and the response rate was $37 \%$, the generalizability of our findings to other femaleonly institutions of higher education may be limited.

\section{Conclusion}

In large 2012 national survey of colleges, $47.6 \%$ of undergraduate students reported that they had not received information regarding depression or anxiety from their academic institution, while $49.0 \%$ reported interest in receiving this information [1]. Given the increasing prevalence of depressive symptoms among college students, systematic screening for mental health concerns at college health centers should be prioritized as a public health issue [20]. Systematic screening that incorporates measures of sleep quality and quantity may improve detection of depression and other mental health disorders [41]. Further, sleep interventions may help control and prevent symptoms of these disorders [42].

Our findings suggest that estimates of current depression prevalence among female US students attending a women's college are similar to those of current depression prevalence among female US coeducational college students. Our findings support the importance of providing undergraduate mental health resources and the need for improved knowledge of the relationships between social support, sleep quality, and depression.

\section{Competing interests}

The authors indicate that they have no financial or other competing interests to declare.

\section{Authors' contributions}

KTW performed statistical analyses and drafted the tables. AEB, AA, DYD, and DMJ performed descriptive statistics. MJM led data collection and analyses. All authors contributed to conception and design of the study, data collection, and drafting the manuscript. All authors read and approved the final manuscript.

\section{Acknowledgements}

The authors would like to acknowledge Dr. Dawn Comeau for her comments on the manuscript. No funding sources were used in the study design, data collection, or the manuscript preparation.

\section{Author details \\ 'Agnes Scott College Department of Public Health, 141 East College Avenue, Decatur, USA. ${ }^{2}$ University of Minnesota School of Public Health, Division of Epidemiology \& Community Health, 420 Delaware Street Southeast, Minneapolis, USA. ${ }^{3}$ Emory University Rollins School of Public Health, Department of Epidemiology, 1518 Clifton Road Northeast, Atlanta, USA.}

Received: 2 September 2013 Accepted: 7 January 2014

Published: 13 January 2014

\section{References}

1. ACHA: American College Health Association-National College Health Assessment II: Undergraduate Students: Reference Group Data Report Spring 2012. Hanover, MD: American College Health Association; 2012.
2. Hysenbegasi A, Hass SL, Rowland CR: The impact of depression on the academic productivity of university students. J Ment Health Policy Econ 2005, 8(3):145-151.

3. Heiligenstein E, Guenther G, Hsu K, Herman K: Depression and academic impairment in college students. J Am Coll Health 1996, 45(2):59-64.

4. Geisner IM, Mallett K, Kilmer JR: An examination of depressive symptoms and drinking patterns in first year college students. Issues Ment Health Nurs 2012, 33(5):280-287.

5. Arria AM, O'Grady KE, Caldeira KM, Vincent KB, Wilcox HC, Wish ED: Suicide ideation among college students: a multivariate analysis. Arch Suicide Res 2009, 13(3):230-246.

6. Farabaugh A, Bitran S, Nyer M, Holt DJ, Pedrelli P, Shyu I, Hollon SD, Zisook $\mathrm{S}$, Baer L, Busse W, et al: Depression and suicidal ideation in college students. Psychopathology 2012, 45(4):228-234.

7. Adams TB, Wharton CM, Quilter L, Hirsch T: The association between mental health and acute infectious illness among a national sample of 18- to 24-year-old college students. J Am Coll Health 2008, 56(6):657-663.

8. Meilman PW, Manley C, Gaylor MS, Turco JH: Medical withdrawals from college for mental health reasons and their relation to academic performance. J Am Coll Health 1992, 40(5):217-223.

9. Conn VS: Depressive symptom outcomes of physical activity interventions: meta-analysis findings. Ann Behav Med 2010, 39(2):128-138.

10. Patten SB, Williams JV, Lavorato DH, Eliasziw M: A longitudinal community study of major depression and physical activity. Gen Hosp Psychiatry 2009, 31(6):571-575.

11. Strohle A: Physical activity, exercise, depression and anxiety disorders. J Neural Transm 2009, 116(6):777-784.

12. Regestein Q, Natarajan V, Pavlova M, Kawasaki S, Gleason R, Koff E: Sleep debt and depression in female college students. Psychiatry Res 2010, 176(1):34-39.

13. Lund $H G$, Reider BD, Whiting $A B$, Prichard JR: Sleep patterns and predictors of disturbed sleep in a large population of college students. J Adolesc Health 2010, 46(2):124-132.

14. Trockel MT, Barnes MD, Egget DL: Health-related variables and academic performance among first-year college students: implications for sleep and other behaviors. J Am Coll Health 2000, 49(3):125-131.

15. Kenney SR, LaBrie JW, Hummer JF, Pham AT: Global sleep quality as a moderator of alcohol consumption and consequences in college students. Addict Behav 2012, 37(4):507-512.

16. Nadorff MR, Nazem S, Fiske A: Insomnia symptoms, nightmares, and suicidal ideation in a college student sample. Sleep 2011, 34(1):93-98.

17. Yen CF, King BH, Tang TC: The association between short and long nocturnal sleep durations and risky behaviours and the moderating factors in Taiwanese adolescents. Psychiatry Res 2010, 179(1):69-74.

18. Breslau N, Roth T, Rosenthal L, Andreski P: Sleep disturbance and psychiatric disorders: a longitudinal epidemiological study of young adults. Biol Psychiatry 1996, 39(6):411-418.

19. Eisenberg D, Gollust SE, Golberstein E, Hefner JL: Prevalence and correlates of depression, anxiety, and suicidality among university students. Am J Orthopsychiatry 2007, 77(4):534-542.

20. Klein MC, Ciotoli $C$, Chung $\mathrm{H}$ : Primary care screening of depression and treatment engagement in a university health center: a retrospective analysis. J Am Coll Health 2011, 59(4):289-295.

21. Eisenberg D, Hunt J, Speer N: Mental health in american colleges and universities: variation across student subgroups and across campuses. J Nerv Ment Dis 2013, 201(1):60-67.

22. Ahmadi J, Samavatt F, Sayyad M, Ghanizadeh A: Various types of exercise and scores on the Beck Depression Inventory. Psychol Rep 2002, 90(3 Pt 1):821-822.

23. Berg KC, Frazier $P$, Sherr L: Change in eating disorder attitudes and behavior in college women: prevalence and predictors. Eat Behav 2009, 10(3):137-142.

24. National Survey of Student Engagement: Converting Data into Action: Expanding the Boundaries of Institutional Improvement. Bloomington: Indiana University Center for Postsecondary Research; 2003.

25. Smith D, Wolf $L$, Morrison D: Paths to success: factors related to the impact of women's college. J High Educ 1995, 66:245-266.

26. Kim M: Cultivating intellectual development: comparing women-only colleges and coeducational colleges for educational effectiveness. Res High Educ 2002, 43:447-481.

27. Smith D: Women's Colleges and coed colleges: is there a difference for women? J High Educ 1990, 61:181-197.

28. Kinzie J, Thomas A, Pamer M, Umbach P, Kuh G: Women students and coeducational and women's colleges: how do their experiences compare? J Coll Student Dev 2007, 48:145-165. 
29. CDC: Behavioral Risk Factor Surveillance System Survey Questionnaire. Atlanta: US Department of Health and Human Services, Centers for Disease Control and Prevention; 2012.

30. Radloff $L$ : The CES-D scale: a self-report depression scale for research in the general population. Appl Psychol Meas 1977, 1(3):385.

31. Lovibond SH, Lovibond PF, Foundation P: Manual for the Depression Anxiety Stress Scales: School of Psychology. Sydney: University of New South Wales, Psychology Foundation; 1995.

32. Greenland S, Pearl J, Robins JM: Causal diagrams for epidemiologic research. Epidemiology (Cambridge, Mass) 1999, 10(1):37-48.

33. Mackenzie S, Wiegel JR, Mundt M, Brown D, Saewyc E, Heiligenstein E, Harahan B, Fleming M: Depression and suicide ideation among students accessing campus health care. Am J Orthopsychiatry 2011, 81(1):101-107.

34. Herman S, Archambeau OG, Deliramich AN, Kim BS, Chiu PH, Frueh BC: Depressive symptoms and mental health treatment in an ethnoracially diverse college student sample. J Am Coll Health 2011, 59(8):715-720.

35. Pilcher JJ, Ginter DR, Sadowsky B: Sleep quality versus sleep quantity: relationships between sleep and measures of health, well-being and sleepiness in college students. J Psychosom Res 1997, 42(6):583-596.

36. Moo-Estrella J, Perez-Benitez H, Solis-Rodriguez F, Arankowsky-Sandoval G: Evaluation of depressive symptoms and sleep alterations in college students. Arch Med Res 2005, 36(4):393-398.

37. Girgenti AA, Mills MJ, Brooks PR: Sleep patterns and symptoms of depression in college students. Coll Student J 2009, 43(9):S464.

38. Lin N, Ye X, Ensel WM: Social support and depressed mood: a structural analysis. J Health Soc Behav 1999, 40(4):344-359.

39. Hefner J, Eisenberg D: Social support and mental health among college students. Am J Orthopsychiatry 2009, 79(4):491-499.

40. Vandervoort D: Quality of social support in mental and physical health. Curr Psychol 1999, 18:205-221.

41. Bliwise DL, Friedman L, Yesavage JA: Depression as a confounding variable in the estimation of habitual sleep time. J Clin Psychol 1993, 49(4):471-477.

42. Trockel M, Manber R, Chang V, Thurston A, Taylor CB: An e-mail delivered CBT for sleep-health program for college students: effects on sleep quality and depression symptoms. J Clin Sleep Med 2011, 7(3):276-281.

doi:10.1186/1472-6874-14-8

Cite this article as: Wilson et al: Social, behavioral, and sleep characteristics associated with depression symptoms among undergraduate students at a women's college: a cross-sectional depression survey, 2012. BMC Women's Health 2014 14:8.

\section{Submit your next manuscript to BioMed Central and take full advantage of:}

- Convenient online submission

- Thorough peer review

- No space constraints or color figure charges

- Immediate publication on acceptance

- Inclusion in PubMed, CAS, Scopus and Google Scholar

- Research which is freely available for redistribution 